\title{
Planetary Nebulae and their parent stellar populations. Tracing the mass assembly of M87 and Intracluster light in the Virgo cluster core
}

\author{
Magda Arnaboldi ${ }^{1,2}$, Alessia Longobardi ${ }^{3}$ and Ortwin Gerhard ${ }^{3}$ \\ ${ }^{1}$ ESO, K. Schwarzschild Str. 2, 85748 Garching, Germany \\ email: marnabol@eso.org \\ ${ }^{2}$ INAF, Oss. Astr. di Pino Torinese, 10025 Pino Torinese, Italy \\ ${ }^{3}$ Max-Planck-Institut für Extraterrestrische Physik, Postsach 1312, 85741 Garching, Germany
}

\begin{abstract}
The diffuse extended outer regions of galaxies are hard to study because they are faint, with typical surface brightness of $1 \%$ of the dark night sky. We can tackle this problem by using resolved star tracers which remain visible at large distances from the galaxy centers. This article describes the use of Planetary Nebulae as tracers and the calibration of their properties as indicators of the star formation history, mean age and metallicity of the parent stars in the Milky Way and Local Group galaxies. We then report on the results from a deep, extended, planetary nebulae survey in a $0.5 \mathrm{deg}^{2}$ region centered on the brightest cluster galaxy NGC 4486 (M87) in the Virgo cluster core, carried out with SuprimeCam@Subaru and FLAMES-GIRAFFE@VLT. Two planetary nebulae populations are identified out to $150 \mathrm{kpc}$ distance from the center of M87. One population is associated with the M87 halo and the second one with the intracluster light in the Virgo cluster core. They have different line-of-sight velocity and spatial distributions, as well as different planetary nebulae specific frequencies and luminosity functions. The intracluster planetary nebulae in the surveyed region correspond to a luminosity of four times the luminosity of the Large Magellanic Cloud. The M87 halo planetary nebulae trace an older, more metal-rich, parent stellar population. A substructure detected in the projected phase-space of the line-ofsight velocity vs. major axis distance for the M87 halo planetary nebulae provides evidence for the recent accretion event of a satellite galaxy with luminosity twice that of M33. The satellite stars were tidally stripped about $1 \mathrm{Gyr}$ ago, and reached apocenter at a major axis distance of $60-90 \mathrm{kpc}$ from the center of M87. The M87 halo is still growing significantly at the distances where the substructure is detected.
\end{abstract}

Keywords. Stars: AGB and post-AGB. (ISM) Planetary nebulae: general. Galaxies: general, abundances, Virgo cluster, elliptical and lenticular, cD, halos, formation, NGC 4486, kinematics and dynamics, structure.

\section{Introduction}

Accretion events are believed to be responsible for the build up of stellar halos in elliptical galaxies (Delucia \& Blaizot 2007) at relatively low redshift $(z<2$; Oser et al. 2010). In the dynamical centers of galaxy clusters, brightest cluster galaxies (BCGs) are expected to have the majority of their stars accreted (Cooper et al. 2015). The galaxy NGC 4486 (M87) is one of the nearest BCGs (at D=14.5 Mpc) in the densest region of the Virgo cluster (Binggeli et al. 1987). Its halo represents a benchmark for studies of the assembly history of extended halos in high density environments. Because of the large orbital time in their outer regions, these halos may still contain fossil records of the mass accretion events that lead to their hierarchical build up. 
M87 has been the target of several imaging surveys and its close proximity made it possible to identify planetary nebulae $(\mathrm{PNs})$ with 8 meter class telescopes (Arnaboldi et al. 2003, Aguerri et al. 2005, Castro-Rodriguez et al. 2009). The goal is to use PN as kinematic tracers and their general $\mathrm{PN}$ population properties as probes for star formation history, age and metallicity of the parent stellar population in those regions of M87 where the surface brightness is too low to carry out absorption line spectroscopy.

In the next sections, we present a concise summary of the general characteristics of PN population as tracers of stars and motions in galaxies and then describe the results from the extended PN survey in M87.

\section{General properties of $P N$ population}

PNs are the final evolutionary stage for most stars in the mass range $1-8 \mathrm{M}_{\odot}$. In the Milky Way (MW), about $95 \%$ of the stars will end their lives as PNs, while only $5 \%$ explode as supernovae. The PN phase lasts $\tau_{P N} \sim 3 \times 10^{4}$ years at most, and its duration depends on the age and metallicity of the parent stellar population (Buzzoni et al. 2006). $\tau_{P N}$ is also related to the expansion time of a nebular shell $\tau_{P N}=D_{P N} / V_{\text {exp }}$, where $D_{P N}$ is the diameter and $V_{\text {exp }}$ is the expansion velocity of a PN shell; typical expansion velocities for the brightest PNs are in the range $11-22 \mathrm{kms}^{-1}$ (Arnaboldi et al. 2008). $\tau_{P N}$ could be shortened by the presence of a hot interstellar medium (Dopita et al. 2000, Villaver \& Stanghellini 2005) which may remove the gaseous shell during its expansion.

Because the diffuse nebula around the core is very efficient in re-emitting $\sim 15 \%$ of the UV energy radiated by the central star in the optical Oxygen forbidden line [OIII] at $5007 \AA$ [which is the brightest optical emission of a PN (Dopita et al. 1992)], PN stars can be efficiently selected via narrow band imaging centered on the Oxygen line.

There are about 2000 PNs known out of 200 billion stars in the MW, and they are mostly concentrated towards the MW plane. A typical Galactic PN has an average shell diameter of about $0.3 \mathrm{pc}$. Hence when a sample of PNs similar to those in the MW are detected in external galaxies at distances larger than $1 \mathrm{Mpc}$, they are identified as spatially unresolved emissions of monochromatic green light at $5007 \AA$.

The integrated [OIII] flux $F_{5007}$ of a spatially unresolved PN can be expressed as $m_{5007}$ magnitude via the formula:

$$
m_{5007}=-2.5 \log \left(F_{5007}\right)-13.74
$$

(Jacoby 1989). Narrow band imaging of external galaxies provide $m_{5007}$ magnitudes for the entire PN population of the surveyed galaxy, down to a given limiting flux. It is then possible to derive the Planetary Nebulae luminosity function (PNLF) $N\left(m_{5007}\right)$ for that PN population at the galaxy distance. The PNLF has been used extensively as secondary distance indicator in early and late-type galaxies within 10-15 Mpc distance (see Ciardullo et al. (2002) for a review). The PNLF is often approximated by an analytical formula given by

$$
N(M) \propto e^{0.307 M} \times\left(1-e^{3\left(M^{*}-M\right)}\right)
$$

as introduced by Ciardullo et al. (1989), where $M^{*}=-4.51$ is the absolute magnitude of the bright cut-off of the PNLF (Ciardullo et al. 1998). This analytical formula is the product of two exponential terms: the first term can be thought of as the dimming of the [OIII] flux as the shell expands at uniform speed (Heinze \& Westerlund 1963), and the second term models the cut-off at bright magnitudes. Hence the formula in Eq. 2.2 describes a PN population as an ensemble of diffuse expanding shells powered 
by unevolving massive cores, all at about $M_{\text {core }} \simeq 0.7 M_{\odot}$, which are emitting a total luminosity of $L \simeq 6000 \mathrm{~L}_{\odot}$ (Ciardullo et al. 2002).

Simple stellar population theory predicts that PN cores should become fainter as the stellar population ages, with core masses as low as $M_{\text {core }} \leqslant 0.55 M_{\odot}$ in a 10 Gyr old, solar-enriched, stellar population (Buzzoni et al. 2006). Marigo et al. (2004) computed a relative dimming of 4 magnitudes for the bright cut-off $M^{*}$ of the PNLF for a 10 Gyr old population with respect to that of a 1 Gyr old population. Such a strong dependence of $M^{*}$ on age is not observed though: empirically, the absolute magnitude of the PNLF bright cut-off is the same for PN populations in star forming disks and in ellipticals (Longobardi et al. (2013)). A possible explanation is that binary stars are progenitors to the brightest PNs in old populations (Ciardullo et al. 2005). Still there may be systematic variations of the PNLF that correlate with the star formation history, mean age and metallicity of the parent stellar population, which can be used to constrain their values and any spatial variations in extended stellar halos.

\subsection{PN visibility lifetime and luminosity functions in the Milky Way and Local Group galaxies}

PN specific frequency - The total number of PNs associated with the bolometric luminosity of a parent stellar population is expressed as $N_{P N}=\alpha L_{\odot, b o l}$, where $\alpha$ is the PN specific frequency. The value of the $\alpha$ parameter is related to the normalization in Eq. 2.2; i.e. to the observed total number of PNs of a given detected population. The value of $\alpha$ is related to the PN visibility lifetime $\tau_{P N}$ by the equation

$$
\alpha=\frac{N_{P N}}{L_{\odot, b o l}}=\mathrm{B} \tau_{P N}
$$

where B (independent of the stellar population) is the $\mathrm{PN}$ formation rate (stars $/ \mathrm{yr} / L_{\odot}$; Buzzoni et al. 2006). The measured values for the $\alpha$ parameter show strong scatter for stellar populations redder than $(B-V) \geqslant 0.8$ (Coccato et al. 2009, Cortesi et al. 2013) with an inverse correlation with the far ultraviolet (FUV) color excess, that is stellar populations with a strong FUV excess are $P N$-starved. Stellar populations with an UV up-turn or FUV color excess are the old and metal rich populations in massive elliptical galaxies. Differently, stellar populations in irregular galaxies like the LMC are PN-rich. In general PN populations show systematic variations of the $\alpha$ values with the integrated photometric properties of the parent stellar population, hence variations of the measured $\alpha$ values as function of radius in an extended stellar halo can be used as a signal for different stellar populations in the halo.

PNLF morphology - The PNLF shows systematic variations that correlate with the average age and metallicity of the parent stellar population. In the $\log (N)$ vs. $m_{5007}$ plot, the gradient of the PNLF within 2.5 magnitude below the brightest can be steeper or shallower than 0.307 (as in Eq. 2.2). The PN population associated with the MW bulge has a steeper PNLF than that derived for the M31 PNs (Arnaboldi et al., in prep.), while those in the outer regions of star-forming disks have a shallower or no gradient (Ciardullo et al. 2004). Longobardi et al. (2013) proposed a generalized formula for the PNLF according to the equation

$$
N(M)=c_{1} e^{c_{2} M} \times\left(1-e^{3\left(M^{*}-M\right)}\right) ; M^{*}=-4.51
$$

where $c_{1}$ is related to the value of the $\alpha$ parameter to first order and $c_{2}$ is related to the gradient of the $\log (N)$ at faint magnitudes. We are planning to apply the generalized formula to complete and extended PN populations and to correlate the $c_{1}, c_{2}$ derived values 


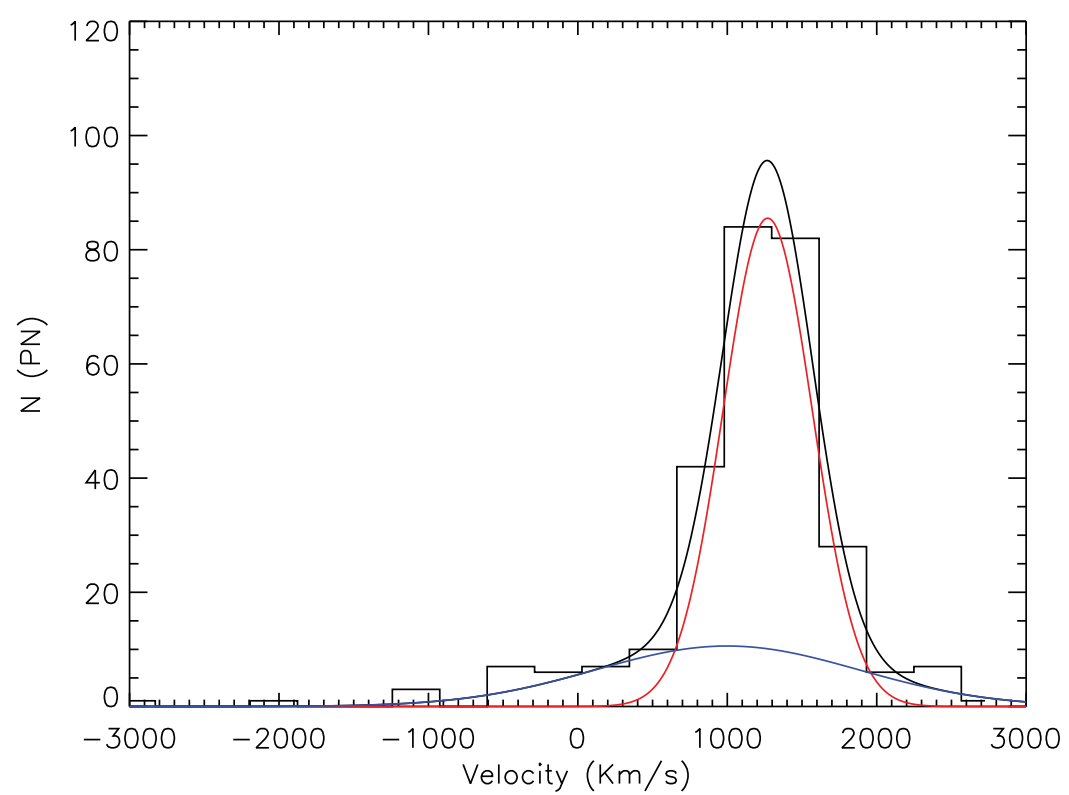

Figure 1. Histogram of the line-of-sight velocities of the spectroscopically confirmed PNs (black histogram) fitted with a double Gaussian (black curve). Red and blue lines represent the two Gaussian associated with the M87 halo and the IC components. From Longobardi et al. (2015a).

with the mean ages and metallicities of the parent stellar populations from spectroscopic measurements, so that we can have a better calibration of the PN probes.

In addition to the gradient, the PNLF of stellar population in low luminosity - metal poor galaxies - shows the presence of a dip. This feature of the PNLF is measured with high significance in the well sampled PNLF for the LMC (Reid \& Parker 2010), the SMC (Jacoby \& De Marco 2002), NGC 6822 (Hernández-Martínez \& Peña 2009) and in the outer regions of M33 (Ciardullo et al. 2004). This dip falls within an interval of 2 to 4 magnitudes below $M^{*}$, but the magnitude at which the dip is detected varies between the PN populations sampled in the Local Group.

Strategy - we can use the global properties of the PN populations, their PNLFs, the gradients, dips and $\alpha$ values to signal transition from old/metal-rich to fading/metal-poor populations when the individual stars cannot be resolved or their surface brightness is too low to carry out integrated light photometry or absorption line spectroscopy.

\section{The PN populations in the Virgo cluster core}

In 2010 we started an imaging survey with SuprimeCam@Subaru to cover $0.5 \mathrm{deg}^{2}$ in the M87 halo; at the distance of the Virgo cluster $(\mathrm{D}=14.5 \mathrm{Mpc})$ this is equivalent to an area of $(130 \mathrm{kpc})^{2}$. We wanted to use the general properties described in Sec. 2 to study its stellar population and kinematics.

We acquired deep narrow band images centered on the [OIII] emission redshifted to the systemic velocity of M87 $\left(V_{\text {sys }}=1275 \mathrm{kms}^{-1}\right)$ and deep off-band images in the V-band. We identified PN candidates as spatially unresolved [OIII] sources with no continuum following the procedure described in Arnaboldi et al. (2002). The final magnitude limited catalog consisted of $688 \mathrm{PN}$ candidates down to 2.5 magnitude from the brightest PN (Longobardi et al. 2013). We then carried out the spectroscopic follow-up with 
FLAMES/GIRAFFE@VLT to acquire spectra for these candidates. We obtained spectra for 289 confirmed PNs (Longobardi et al. 2015a) which we analyzed together with 12 previous identified PNs from Doherty et al. (2009), for a total combined sample of 301 PNs.

We built the line-of-sight velocity distribution (LOSVD) for the whole PN sample: this is shown in Fig. 1. The LOSVD is characterized by a strong peak at $1275 \mathrm{kms}^{-1}$ and a second moment $\sigma_{n} \simeq 300 \mathrm{kms}^{-1}$, with large asymmetric wings, with a tail extended towards zero and negative LOS velocities. These wings can be modeled with a broad Gaussian component, centered at $v_{b}=995 \mathrm{kms}^{-1}$ and $\sigma_{b}=900 \mathrm{kms}^{-1}$. The narrow component is consistent with the LOSVD for the stars in the M87 halo, while the broad component has a LOSVD similar to that of Virgo cluster galaxies. We thus selected the M87 halo PNs as those associated with the narrow component in the LOSVD by means of a robust iterative procedure (Longobardi et al. 2015a). The PNs in the broad asymmetric wings of the LOSVD are thereby tagged as Virgo intracluster (IC) PNs. In Figure 2 we show the projected phase space diagram for the $301 \mathrm{PNs}$ around M87 and the additional ICPNs from Doherty et al. (2009).

We studied the spatial distribution of the two selected PN samples and compared their profiles with the V band photometry of M87 by Kormendy et al. (2009). The number density profile of the M87 halo PNs follows the Sersic $n=11.8$ surface brightness profile, while that of the ICPNs follows a much flatter radial profile, which is consistent with a power law, $N_{I C P N}(R) \propto R^{\gamma}$ with $\gamma=[-0.34,-0.04]$. By scaling the M87 PN density profile to the inner regions we derived the value of the $\alpha$ parameter for the M87 PN halo population: $\alpha_{\text {halo }}=(1.06 \pm 0.12) \times 10^{-8} P N L_{\odot, b o l}^{-1}$. The value of the $\alpha$ parameter for the intracluster light is different: the same procedure applied to the ICPN number density profile returns $\alpha_{I C L}=(2.72 \pm 0.72) \times 10^{-8} P N L_{\odot, b o l}^{-1}$.

We then compared our $\alpha_{\text {halo }}$ and $\alpha_{I C L}$ values with those for PN populations in nearby galaxies. Galaxies with $(B-V) \leqslant 0.8$ have $\mathrm{PN}$ specific frequency values similar to that of $\alpha_{I C L}$. For redder galaxies, the scatter increases; the value of $\alpha_{\text {halo }}$ is the same as for populations with an FUV excess that are PN-starved. Hence the IC component contributes three times more PN per unit bolometric luminosity than the M87 halo light, signaling a change of population from halo to ICL. This transition is consistent with the existence of a color gradient towards bluer colors in M87 at large radii (Rudick et al. 2010 ), and an ICL population that is mostly old (age $\simeq 10$ Gyr) with a mean metallicity of $[M / H] \simeq-1.0$ (Williams et al. 2007). On the basis of the kinematic separation of the M87 halo and intracluster PNs, we built the two independent PNLFs, and examined them in turn. The PNLF for the M87 halo PNs is steeper than in Eq. 2.2. We used the generalized formula from Eq. 2.4 and derived $c_{2}=0.72$ and a distance modulus of $m-M=30.8$. The PNLF of the ICPNs shows a dip at $1-1.5 \mathrm{mag}$ below $M^{*}$, reminiscent of the morphology of the PNLF observed in PN populations associated with low luminosity/metal poor galaxies. We fitted the generalized PNLF formula to the PNLF of the ICPNs, and found $c_{2}=0.66$ and a distance modulus of $m-M=30.8$.

We could estimate the luminosity associated with the ICPNs by integrating the ICL surface brightness profile over the surveyed area, which amounts to $L_{I C L}=5.3 \times$ $10^{9} L_{\odot, b o l}$. From the presence of the "dip" of the PNLF, the parent stellar population is similar to that of the Magellanic Clouds or in the outer regions of the M33 disk. Thus the sampled luminosity of the ICL is equivalent to four time the luminosity of the $L M C$ or 1.5 times that of $M 33$, over the whole surveyed area of $(130 \mathrm{kpc})^{2}$. 


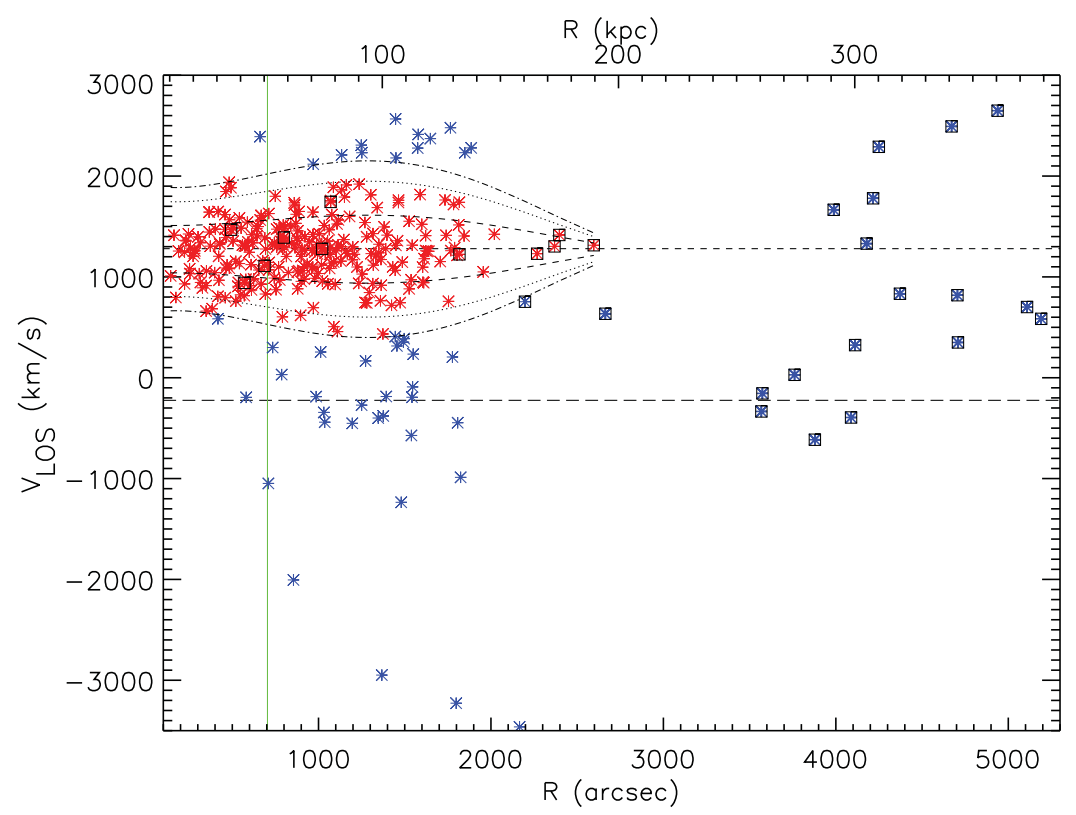

Figure 2. Projected phase-space diagram, $V_{L O S}$ vs. major axis distance from the center of M87, for all spectroscopically confirmed PNs in the Virgo core.The major axis distance is given both in arcsec (bottom axis), and in kpc (top axis), where $73 \mathrm{pc}=1^{\prime \prime}$. The PNs are classified as M87 halo PNs (red asterisks) and ICPNs (blue asterisks), respectively. Black squares identify spectroscopically confirmed PNs from Doherty et al. (2009). The dashed horizontal line shows the M87 systemic velocity $V_{s y s}=1275 \mathrm{kms}^{-1}$, while the continuous green line shows the effective radius $R_{e}=703.914^{\prime \prime}$ (Kormendy et al. 2009). From Longobardi et al. (2015a).

\section{The late mass assembly of the M87 halo}

We then turned to the M87 halo PNs and studied the projected phase-space diagram $v_{L O S}$ vs. $R_{M a j}$ for this component. The density of points is not uniform in the range of velocities covered by the M87 halo. There is a clearly identifiable $\mathbf{V}$-shaped over-density, or "Chevron", between 30 and $90 \mathrm{kpc}$ radii, with its vertex, or edge, culminating at 90 kpc or $1200^{\prime \prime}$ (Longobardi et al. 2015b). These high density substructures in phase-space are likely to be associated with the disruption of a satellite galaxy in the deeper potential of a massive host (Quinn 1984).

We looked at the LOSVD in three radial bins over the radial range of the Chevron, and employed a Gaussian Mixture Model to assign each M87 halo PN a probability to belong to the $\mathbf{V}$ substructure or to the smooth halo. A total of $54 \mathrm{PNs}$ are thereby associated to the $\mathbf{V}$ substructure and $200 \mathrm{PNs}$ to the smooth halo component (Longobardi et al. 2015b). We then looked at the spatial distribution of the Chevron PNs: the highest density occurs at $1200^{\prime \prime}$ NW along the M87 major axis, and is spatially correlated with a diffuse, extended substructure, labeled "the crown" of M87 (Longobardi et al. 2015b), see Figure 3. The luminosity of the substructure is about $60 \%$ of the light at the location where the crown is found. We computed the total luminosity of the disrupted satellite from the 54 PNs: it amounts to $L_{\text {sat }}=2.8 \times 10^{9} L_{\odot, b o l}$. We also looked at correlation between the high density of the chevron PNs and the (B-V) color in the M87 halo. A strong correlation is found, with bluer color $(\mathrm{B}-\mathrm{V}=0.76)$ measured at the position of the "crown". From the luminosity and the color we infer that the luminosity of the dissolved satellite is equivalent to about twice the luminosity of M33. 

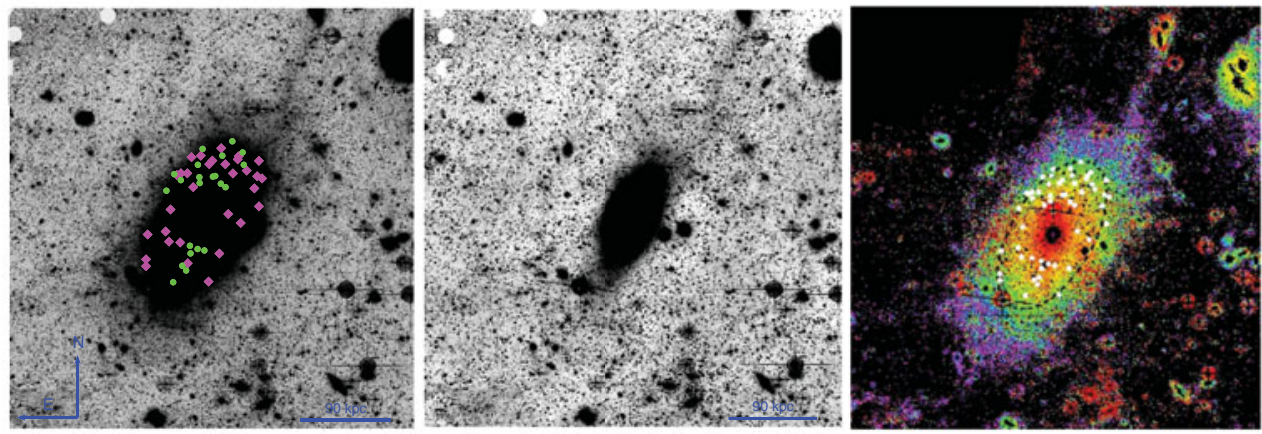

Figure 3. Spatial and color distribution associated with the kinematic substructure identified in the phase space of the M87 halo PNs. Left panel: V-band image of a $1.6 \times 1.6 \mathrm{deg}^{2}$ centered on M87 from Mihos et al. (in prep.). Full circles, and diamonds indicate the spatial positions of the M87 halo PNs in the chevron substructure. Magenta and green colors indicate PN LOSVs above and below $V_{L O S}=1254 \mathrm{kms}^{-1}$, the LOSV at the end of the Chevron. Central panel: unsharp masked image of M87 median binned to enhance faint structures. The crown-shaped substructure is visible at distance of $800^{\prime \prime}-1200^{\prime \prime}(60-90 \mathrm{kpc})$ along the major axis, NW of M87. The blue line measures $90 \mathrm{kpc}$. Right panel: $(\mathrm{B}-\mathrm{V})$ color image of M87 from Mihos et al. (in prep.) with chevron PNs overplotted (white dots). The dashed ellipse indicates the isophote at a major axis distance of $1200^{\prime \prime}$. The crown is found in a region where the $(\mathrm{B}-\mathrm{V})$ color is on average 0.8 , bluer than on the minor axis. From Longobardi et al. (2015b).

From the distribution and velocities of Chevron PNs in Figure 3, a possible interpretation of the satellite orbit could be that it was first disrupted entering M87 from the south (along the green dots), with the debris then moving up north, turning around in the crown region, coming back down on both sides across M87 (magenta dots). The velocities would then imply that the northern side of M87 is closer to the observer. The dynamical time for such an orbit is of the order of 1 Gyr (Weil et al. 1997).

\section{Conclusions}

Using PNs as tracers we showed that the stellar halo of the BCG M87 is distinct from the surrounding ICL in its kinematics, density profile, and parent stellar population, consistent with the halo of M87 being redder and more metal-rich that the ICL. The ICL in our surveyed fields corresponds to about four times the luminosity of the LMC, spread out over a region of $(130 \mathrm{kpc})^{2}$. It is remarkable that population properties can be observed for such a diffuse component. Based on its population properties we propose that the progenitors of the Virgo ICL were low-mass star forming galaxies.

We also presented kinematic and photometric evidence for an accretion event in the halo of M87. This event is traced by PNs whose phase space shows a distinct chevron-like feature, which is the result of the incomplete phase-space mixing of a disrupted galaxy. At a major axis distance of $R \sim 69-90 \mathrm{kpc}$ where the width of the chevron goes to zero, a deep optical image shows the presence of a crown-like substructure that contribute more than $60 \%$ of the light in this area. The luminosity of the satellite corresponds to about two times M33 with color $(B-V)=0.76$. The similar colors of the accreted satellite and ICL suggest that the halo of M87 is presently growing through the accretion of similar starforming systems as those that build up the diffuse ICL component. The newly discovered substructure within the halo of M87 demonstrates that beyond a distance of $60 \mathrm{kpc}$ its halo is still assembling. 


\section{Acknowledgments}

The authors wish to thank J.C Mihos, R. Hanuschik for their contribution, and the time allocation committees of the Subaru Telescope and the ESO OPC for the opportunity to carry out this exciting project. Based on observations made with the VLT at Paranal Observatory under programmes 088.B-0288(A) and 093.B-066(A), and with the Subaru Telescope under programme S10A-039.

\section{References}

Aguerri, J. A. L., Gerhard, O. E., Arnaboldi, M., Napolitano, N. R., et al. 2005, AJ, 129, 2585 Arnaboldi, M., Doherty, M., Gerhard, O. E., Ciardullo, R. et al. 2008, ApJ, 674, L17 Arnaboldi, M., Freeman, K. C., Okamura, S., Yasuda, N. et al. 2003, AJ, 125, 514 Arnaboldi, M., Aguerri, J. A. L., Napolitano, N. R., Gerhard, O. E. et al. 2002, AJ, 123, 760 Binggeli, B., Tammann, G. A., \& Sandage, A. 1987, AJ, 94, 251

Buzzoni, A., Arnaboldi, M., \& Corradi, R. 2006, MNRAS, 368, 877

Castro-Rodriguez, N., Arnaboldi, M., Aguerri, J. A. L., Gerhard, O. et al. 2009, A\&্A, 507, 621 Ciardullo, R., Sigurdsson, S., Feldmeier, J. J., Jacoby, G. H. 2005 ApJ 629, 499

Ciardullo, R., Durrell, P. R., Laychak, M. B. et al. 2004, ApJ, 614, 167

Ciardullo, R., Feldmeier, J. J., Jacoby, G. H., Kuzio de Naray, R. et al. 2002, ApJ, 577, 31

Ciardullo, R., Jacoby, G. H., Feldmeier, J. J., \& Bartlett, R. E. 1998, ApJ, 492, 62

Ciardullo, R., Jacoby, G. H., Ford, H. C., \& Neill, J. D. 1989, ApJ, 339, 53

Coccato, L., Gerhard, O., Arnaboldi, M. et al. 2009, MNRAS, 394, 1249

Cooper, A. P., Gao, L., Guo, Q., Frenk, C. S., et al. 2015, MNRAS, 451, 2703

Cortesi, A., Arnaboldi, M., Coccato, L. et al. 2013, A\& $A$, 549, 115

De Lucia, G.,\& Blaizot, J. 2007, MNRAS, 375, 2

Doherty, M., Arnaboldi, M., Das, P., Gerhard, O. et al. 2009, A\&A, 502, 771

Dopita, M., Massaglia, S., Bodo, G., Arnaboldi, M. et al. 2000, ASPC, 199, 423

Dopita, M., Jacoby, G. H., \& Vassiliadis, E. 1992, ApJ, 389, 27

Jacoby, G. H. \& De Marco, O. 2002, AJ, 123, 269

Jacoby, G. H. 1989, ApJ, 339, 39

Kormendy, J., Fisher, D. B., Cornell, M. E., \& Bender, R. 2009, ApJS, 182, 216

Hernández-Martínez, L. \& Peña, M. 2009, A\&A, 495, 447

Henize, K. G. \& Westerlund, B. E. 1963, ApJ, 137, 747

Longobardi, A., Arnaboldi, M., Gerhard, O., \& Hanuschik, R. 2015a, A\&A 579, 135

Longobardi, A., Arnaboldi, M., Gerhard, O., \& Mihos, J. C. 2015b, A\&GA 579L, 3

Longobardi, A., Arnaboldi, M., Gerhard, O., Coccato, L. et al. 2013, A\&A 558, 42

Marigo, P., Girardi, L., Weiss, A., Groenewegen, M. A. T., et al. 2004, A\& A, 423,

Oser, L., Ostriker, J. P., Naab, T., Johansson, P. H., et al. 2010, ApJ, 725, 2312

Quinn, P. J. 1984, ApJ, 279, 596

Reid, W. A. \& Parker, Q. A. 2010, MNRAS, 405, 1349

Rudick, C. S., Mihos, J. C., Harding, P., Feldmeier, J. J. et al. 2010, ApJ, 720, 569

Villaver, E. \& Stanghellini, L. 2005, ApJ, 632, 854

Weil, M. L., Bland-Hawthorn, J., \& Malin, D. F. 1997, ApJ 490, 664

Williams, B. F., Ciardullo, R., Durrell, P. R., Vinciguerra, M. et al. 2007, ApJ, 656, 756 